\title{
Triple-negative breast cancer: clinicopathological characteristics and relationship with basal-like breast cancer
}

\author{
Aye Aye Thike ${ }^{1}$, Poh Yian Cheok ${ }^{1}$, Ana Richelia Jara-Lazaro ${ }^{1}$, Benita Tan ${ }^{2}$, Patrick Tan ${ }^{3,4}$ \\ and Puay Hoon Tan ${ }^{1}$ \\ ${ }^{1}$ Department of Pathology, Singapore General Hospital, Singapore; ${ }^{2}$ Department of General Surgery, \\ Singapore General Hospital, Singapore; ${ }^{3}$ Duke-NUS Graduate Medical School, Singapore and ${ }^{4}$ Genome \\ Institute of Singapore, Singapore
}

\begin{abstract}
Triple-negative breast cancer, defined as that with negative expression of estrogen and progesterone receptors and cerbB2, accounted for $11 \%$ of invasive breast cancers in our study, drawn from an original cohort of 7048 women diagnosed with breast cancer from the files of the Department of Pathology, Singapore General Hospital, over 14 years. Women with triple-negative breast cancer were generally postmenopausal, with adverse pathological characteristics of high histological grade and frequent nodal metastases. Using a set of 61 invasive breast cancers earlier profiled into molecular subtypes with expression arrays, we defined specificity and sensitivity values for different immunohistochemical panels of basal keratins (CK5/6, CK14, CK17, 34 $\beta E 12)$, CD117, EGFR, p63 and SMA in defining basal-like breast cancer. Subsequent application of a tri-panel of CK14, EGFR and 34/BE12 (specificity $100 \%$ and sensitivity $78 \%$ ) to our group of 653 triple-negative breast cancers delineated $84 \%$ to be basal-like. Immunohistochemical expression of individual biological markers correlated with unfavorable pathological parameters. We conclude that triple-negative breast cancers in an Asian population harbor adverse pathobiological features, and an immunohistochemical surrogate panel can be reliably used to define basal-like cancers among them.

Modern Pathology (2010) 23, 123-133; doi:10.1038/modpathol.2009.145; published online 23 October 2009
\end{abstract}

Keywords: triple negative; basal-like; adverse pathologic parameters; immunohistochemistry; expression profiles

Breast cancer is a heterogeneous disease, which can be characterized into clinically, morphologically and biologically meaningful groups. There has been recent intense interest in the subset of breast cancer referred to as triple-negative breast cancer that lacks the expression of hormone receptors and HER2 (cerbB2). Triple-negative breast cancer has more aggressive clinical behavior, ${ }^{1-4}$ distinctive metastatic patterns $s^{5,6}$ and poor prognosis. ${ }^{7,8}$ Attention on triplenegative breast cancer also relates to the lack of tailored therapies for this group of breast cancer patients, and its overlap with descriptions of basaltype breast cancer, as many triple-negative breast

Correspondence: Dr PH Tan, FRCPA, Department of Pathology, Singapore General Hospital, Outram Road, Singapore 169608, Singapore.

E-mail: gpttph@sgh.com.sg

Received 20 July 2009; revised 3 September 2009; accepted 8 September 2009; published online 23 October 2009 cancers manifest basal characteristics in their molecular profiles. Triple-negative breast cancer accounts for $10-17 \%$ of all breast carcinomas depending on thresholds used to define estrogen receptor (ER) and progesterone receptor (PR) positivity, as well as methods and criteria for HER2 assessment. $^{8-15}$ Both triple-negative and basal-like breast cancers tend to be of high histological grade with limited therapeutic options, and a significant overlap in their biological and clinical characteristics is repeatedly shown by molecular techniques. ${ }^{2,7,16-18}$

The advent of tissue microarrays and highthroughput pathology has placed demands on the quality, reproducibility and accuracy of immunohistochemical assays, many of which have been used to address and develop immunohistochemical surrogates for tumors interrogated through molecular profiling. ${ }^{19}$ It is understandable that immunohistochemistry may be a more appropriate and 
convenient technique in the clinical setting than gene expression-based platforms, in view of cost and equipment availability issues of the latter in routine service laboratories. To date, the true relationship between triple-negative breast cancer and those showing basal-like expression profiles or basal-type proteins (basal-like cancers) is not completely enunciated. There have been reports lumping triple-negative breast cancer with basal-like breast cancers. Yet, there are studies that reveal that not all triple-negative breast cancers express basal characteristics, and hence these two groups of breast cancers are not synonymous. ${ }^{20}$

Our study aimed to pathologically illustrate triple-negative breast cancer in our population, and subject these cancers to a broad panel of biomarkers, including those of a basal nature, to better comprehend the relationship between triplenegative and basal-like breast cancers in our women. In addition, we compared basal protein expression in a subset of molecularly characterized basaloid breast cancers on the basis of expression profiling.

\section{Materials and methods}

\section{Patients and Tumors}

Of the 7048 women diagnosed with primary breast cancer between 1994 and 2007 at the Department of Pathology, Singapore General Hospital, 767 invasive tumors were triple negative according to archival reports that documented ER, PR and cerbB2 negativity. Clinicopathological parameters including age, ethnicity, tumor size, histological grade, nuclear pleomorphism, mitotic score, tubule formation/ score, histological subtype, associated ductal carcinoma in situ, lymphovascular invasion and nodal status were evaluated. Histological grade, nuclear pleomorphism, mitotic score, tubule formation and histological subtype were assessed in accordance with standard guidelines. ${ }^{21}$ Ductal carcinoma in situ was classified according to nuclear grade. Tumor margin, necrosis, degree of lymphocytic infiltrates and growth pattern were reviewed. Tumor margin was considered infiltrating when there were tonguelike protrusions of cancer cells into the surrounding stroma, beyond the general contours of the tumor. A pushing margin was one with generally rounded peripheries (Figure 1). Necrosis was assessed as present or absent (Figure 2). Lymphocytic infiltrates were stratified into mild, moderate and marked, and assessed on the basis of the quantum of lymphocytes present in the tumor (Figure 3). When less than a third of the tumor incorporated lymphocytic infiltrates, a third to two-thirds of the tumor included lymphocytes and more than two-thirds of the tumor harbored lymphocytic accompaniment, they corresponded to mild, moderate and marked lymphocytic infiltrates, respectively. Trabecular growth inferred groups of tumor cells arranged in bars and beams, whereas syncytial growth was defined by tumor
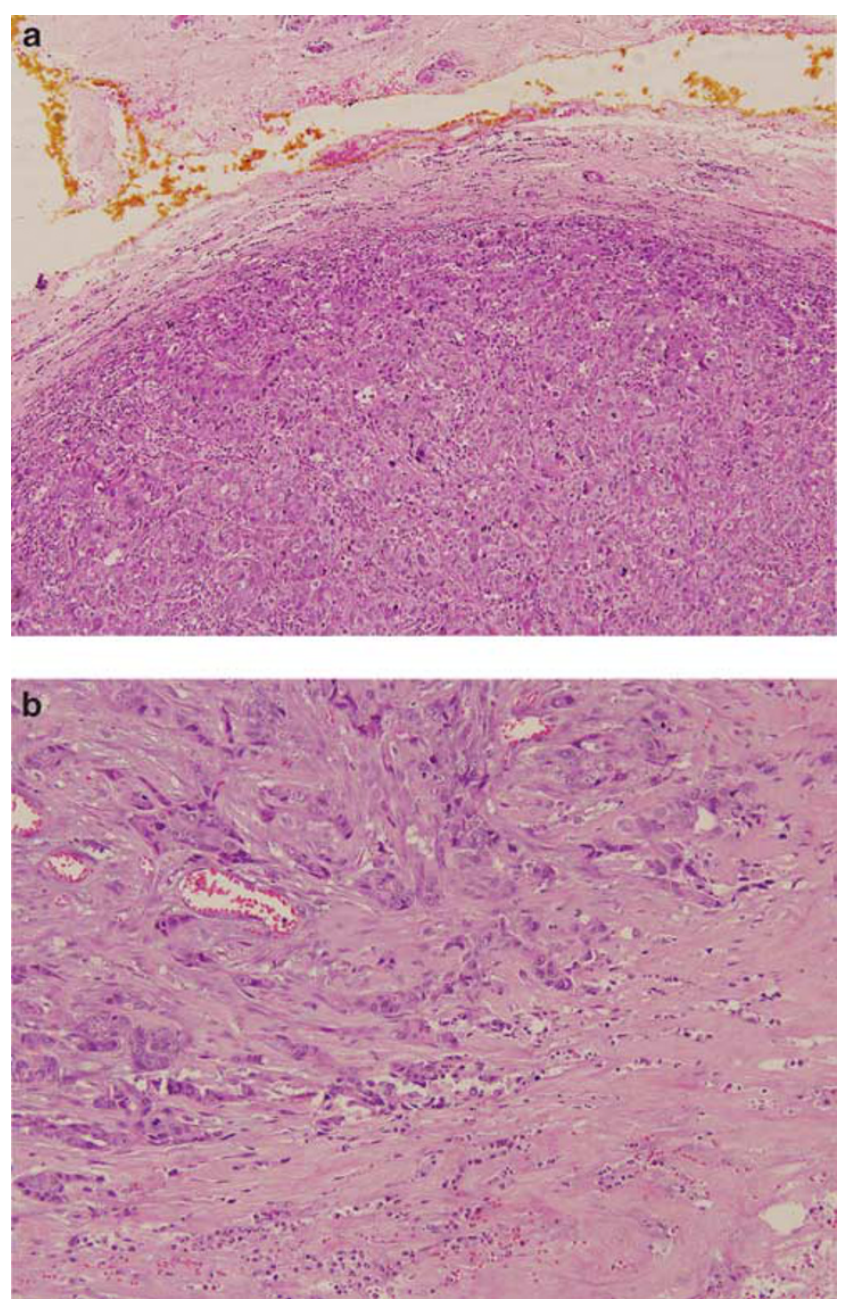

Figure 1 (a) Pushing or rounded margin at which the tumor edge showed a circumscribed boundary. (b) Infiltrative margin at which there were permeative tumor tongues at the advancing tumor front.

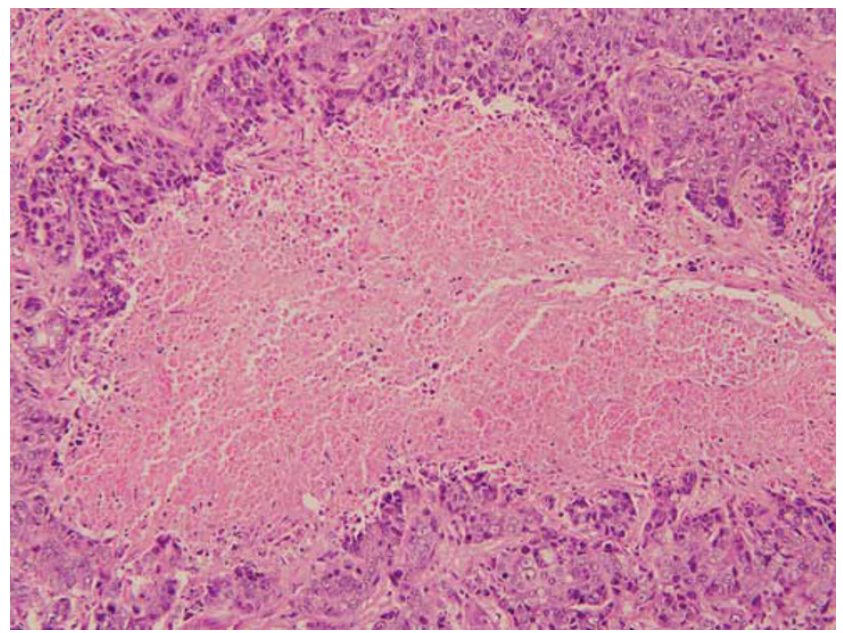

Figure 2 Necrosis observed within the tumor. 

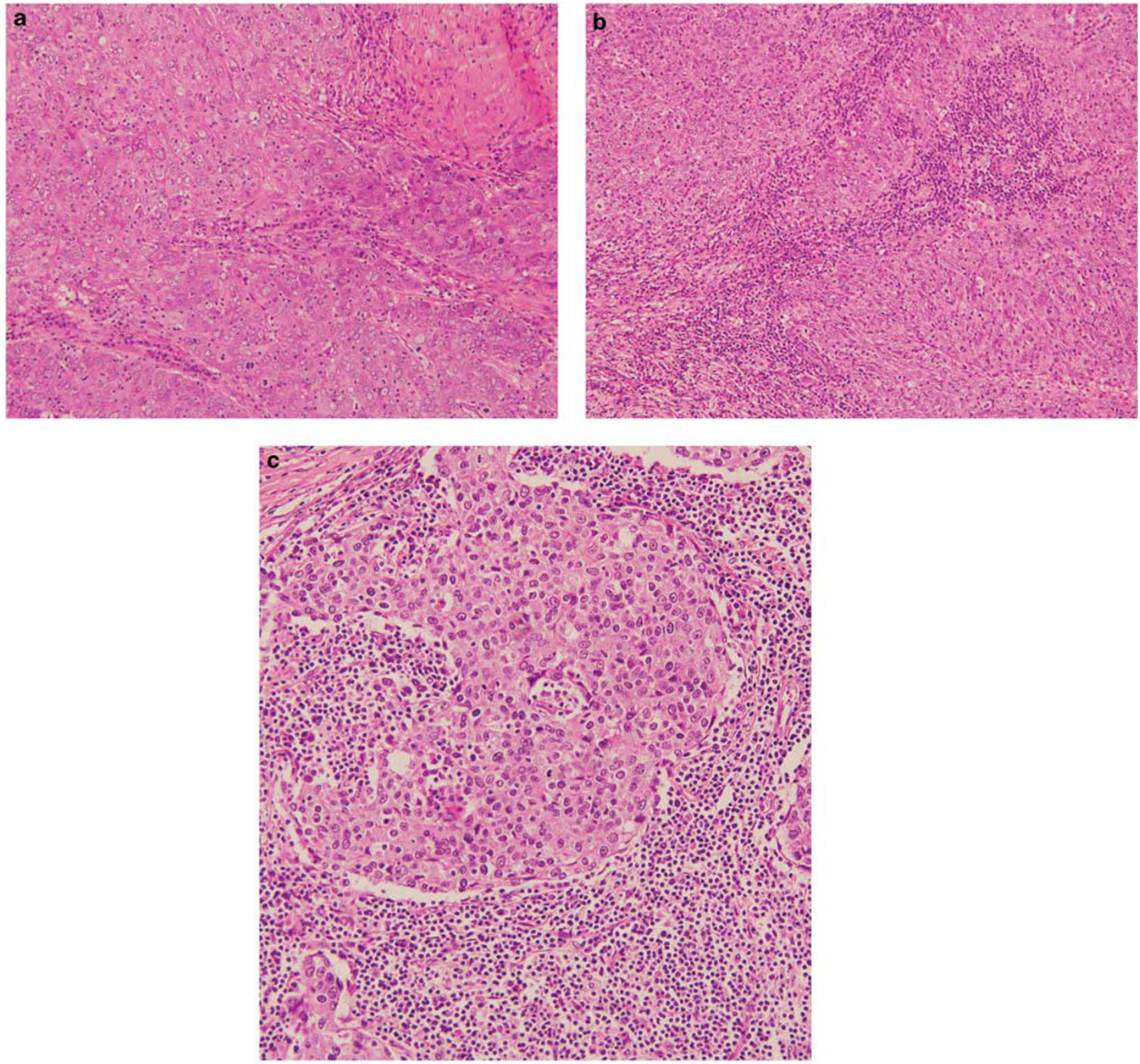

Figure 3 (a) Mild lymphocytic infiltrates with $<1 / 3$ of the tumor area revealing lymphoplasmacytic collections. (b) Moderate lymphocytic infiltrates showing just over $1 / 3$ of the tumor harboring lymphocytes and plasma cells. (c) Marked lymphocytic infiltrates with $>2 / 3$ of the tumor area obscured by lymphocytes and plasma cells.

cells intimately apposed and nestled against one another without distinct cytoplasmic membranes, forming a large syncytium (Figure 4).

\section{Tissue Microarrays and Immunohistochemistry}

Histological slides were retrieved and reviewed, and representative areas were selected for tissue microarray construction, using the Beecher microarrayer with $1 \mathrm{~mm}$ cores and two cores per case. Of the 767 triple-negative breast cancers from our archival files, 40 tumors were diagnosed on core biopsies and in 48 cases, suitable archival paraffin blocks were not available for tissue microarray construction. Finally, a total of 679 triple-negative tumors were subjected to tissue microarray construction.

For immunohistochemistry, $4 \mu \mathrm{m}$ sections were cut from tissue microarray blocks and fished onto coated slides (POLYSINE, Menzel-glaser) in a similar orientation to facilitate evaluation. Sections were restained with anti-ER, anti-PR and anti-cerbB2 antibodies using routine protocols. Briefly, paraffin sections of formalin-fixed tissue were stained for ER using Neomarker RM9101-S with 1:50 dilution, PR using Neomarker RM9102-S with 1:200 dilution and cerbB2 using Neomarker RM9103-S with 1:200 dilution. The sections were pretreated using a 

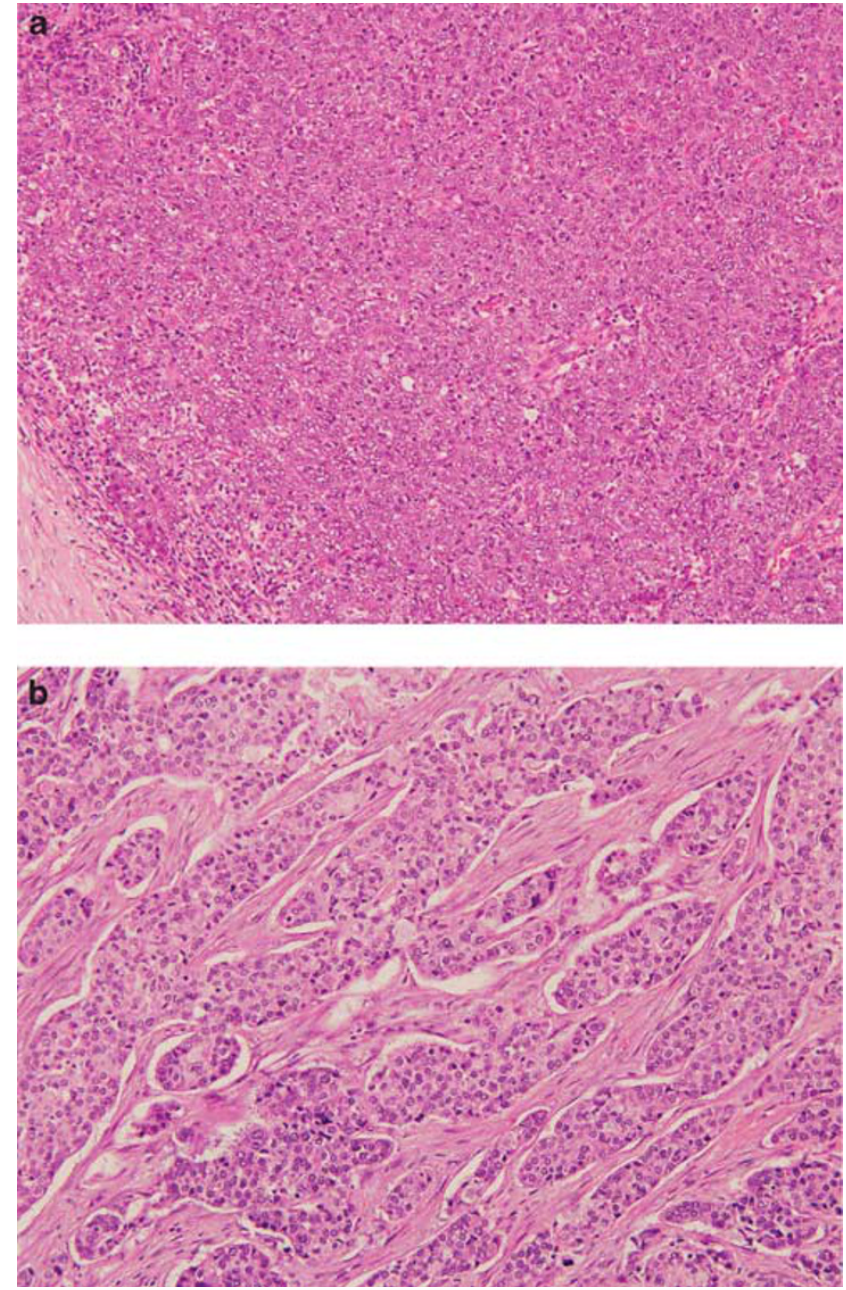

Figure 4 (a) Syncytial growth pattern with sheets of cells intimately apposed without discernible cell membranes. (b) Trabecular growth pattern with broad but discrete tumor beams.

microwave at $98^{\circ} \mathrm{C}$ for $12 \mathrm{~min}$ in Ventana CCI solution (Ventana Medical Systems, Arizona, USA). The detection system used was Linked Streptavidin Biotin. The tissue microarray cores were reviewed and triple negativity was reconfirmed. For ER and PR, a result was considered positive if at least $10 \%$ of tumor cells displayed a minimum of $2+$ nuclear staining. For cerbB2, a test was considered positive if at least $30 \%$ of tumor cells exhibited $3+$ cell membrane staining and a borderline/equivocal result was given when at least $10 \%$ of tumor cells showed $2+$ cytoplasmic membrane staining. The results that failed to fulfill the above criteria were considered negative. Tumors in which any one of the above three markers showed immunoreactivity were excluded from the analysis. Benign breast epithelium served as internal positive controls for ER and PR, whereas a tumor with known cerbB2 overexpression was used as the positive control for cerbB2.

After repeat ER, PR and cerbB2 immunostaining, 10 tumors showed ER positivity, 2 revealed PR
Table 1 Details of antibodies and dilutions

\begin{tabular}{lllc}
\hline Antibody & Clone & Source & Dilution \\
\hline CK5/6 & D5/16 B4 & Dako M7237 & $1: 20$ \\
CK14 & LL002 & NovocastraNCL-LL002 & $1: 20$ \\
CK17 & E3 & Dako M7046 & $1: 20$ \\
CD117 & Ra & Dako A4502 & $1: 200$ \\
EGFR & E30 & Dako M7239 & $1: 50$ \\
p63 & 7JUL & Novocastra NCL-p63 & $1: 100$ \\
SMA & 1A4 & Dako 0851 & $1: 500$ \\
Cytokeratin HMW & 34 $\beta$ E12 & Dako M0630 & $1: 200$ \\
\hline
\end{tabular}

Microwave pretreatment in Tris-EDTA pH 8.7.

positivity, 3 showed both ER and PR positivity, and 11 tumors disclosed cerbB2-positive immunoexpression. All these 26 cases were excluded, with a final 653 triple-negative breast cancers forming the basis of this study.

These 653 triple-negative breast cancers were subjected to antibodies to CK5/6, CK14, CK17, EGFR, CD117, p63, SMA and $34 \beta$ E12, which were applied to sections cut from tissue microarray blocks using routine immunohistochemical protocols. Details of antibodies are shown in Table 1. Cytoplasmic staining for CK5/6, CK14, CK17, SMA, CD117, $34 \beta \mathrm{E} 12$, nuclear reactivity for p63 and cytoplasmic membrane positivity for EGFR were considered positive. Appropriate controls were used. Staining intensity was scored as $0,1+, 2+$ and $3+$ (no staining, weak, moderate and strong staining, respectively). The percentage of positively stained tumor cells was assessed as a proportion of the total number of tumor cells present in the section. Immunoreactive score and intensity percentage score were documented. Immunoreactive score was calculated as follows: $(3 \times \%$ strong staining $)+$ $(2 \times \%$ moderate staining $)+(1 \times \%$ weak staining $)$. Intensity percentage score was defined as the product of the maximum immunostaining intensity and the percentage of tumor cells stained.

Cases of invasive lobular carcinoma were confirmed with negative staining for E-cadherin (Clone NCH-38, Dako M3612, dilution 1:30, with microwave antigen retrieval).

\section{Molecular Subtypes of Breast Cancer}

On the basis of an earlier published study on clinical validation of a customized multiple signature microarray for breast cancer molecular subtypes, including the basaloid subtype, ${ }^{22}$ we performed immunohistochemical protein validation in 61 cases of invasive ductal breast carcinomas which had been classified on expression profiling. These included 28 cases designated as basaloid, 13 cerbB2 and 20 luminal phenotypes on expression arrays. Corresponding tumor paraffin blocks of these cases were subjected to tissue microarray construction and immunohistochemistry as described above. 
Antibodies to ER, PR, cerbB2, basal markers (CK5/6, CK14, CK17, 34ßE12), CD117, EGFR, p63 and SMA were applied to $4 \mu \mathrm{m}$ sections cut from the constructed tissue microarray block and immunohistochemical detection was performed using the Linked Streptavidin Biotin method. Immunoscoring was applied as described.

\section{Statistical Analysis}

Findings were analyzed using statistical software SPSS for Windows, Version 16. The relationship between clinicopathological parameters, immunohistochemical staining intensity, percentage of positively stained tumor cells, immunoreactive score and intensity percentage score of markers was tested using the $\chi^{2}$ and Fisher's exact tests. A $P$-value $<0.05$ defined statistical significance.

Immunohistochemical reactivity of basal and other biological markers in 61 cases of invasive ductal carcinoma earlier characterized on expression profiling was assessed to determine the distribution of cases into true and false-positive, true and false-negative categories. We calculated percent sensitivity as $100 \times$ [true positive/(true positive + false negative)] and specificity as $100 \times$ [true negative/(true negative + false positive)] . To identify criteria providing optimal discrimination, we plotted receiver-operating characteristic curves. We also estimated areas under the receiveroperating characteristic curves using the method of successive parallelograms. ${ }^{23}$ Estimates were considered statistically significant for two-tailed values of $P<0.05$.

\section{Results}

\section{Clinicopathological Features}

Of 7048 women diagnosed with primary breast cancer between 1994 and 2007 at the Department of Pathology, Singapore General Hospital, 1145 $(16 \%)$ were ductal carcinoma in situ, making the total number of invasive breast cancers 5903 (84\%). On the basis of archival pathology reports, 741 of $5903(13 \%)$ cases were triple negative for ER, PR and cerbB2. However, on repeat immunohistochemical evaluation of 679 archivally documented triplenegative breast cancers, 26 cases showed positivity of one or more of these markers. After exclusion of cases that were not retested from the denominator of invasive breast cancers, the proportion of triplenegative breast cancers among our women is finalized as $11 \%$.

Clinicopathological characteristics of 653 triplenegative breast cancers are shown in Table 2 . Ninety-eight $(15 \%)$ patients were 40 years of age or younger. The majority (538/653, 82\%) were Chinese. Triple-negative breast cancers in our study cohort were mostly high grade/grade 3 (503 out of 653,
Table 2 Clinicopathological features of triple-negative breast cancer $(N=653)$

\begin{tabular}{|c|c|}
\hline Clinicopathologic parameters & Number of cases (\%) \\
\hline \multicolumn{2}{|c|}{ Age (years) (mean 53, median 52, range 25-89) } \\
\hline$<40$ & $98(15 \%)$ \\
\hline 40 to 50 & $193(30 \%)$ \\
\hline$>50$ & $355(54 \%)$ \\
\hline Not available & $7(1 \%)$ \\
\hline \multicolumn{2}{|l|}{ Ethnicity } \\
\hline Chinese & $538(82 \%)$ \\
\hline Malay & $55(8 \%)$ \\
\hline Indian & $35(5 \%)$ \\
\hline Others & $23(4 \%)$ \\
\hline Not mentioned & $2(1 \%)$ \\
\hline \multicolumn{2}{|c|}{ Tumor size (mm) (mean 29, median 25, range $0.9-200$ ) } \\
\hline$\leq 20$ & $169(26 \%)$ \\
\hline$>20$ & $460(70 \%)$ \\
\hline Unknown & $24(4 \%)$ \\
\hline \multicolumn{2}{|l|}{ Histologic grade } \\
\hline 1 & $17(2 \%)$ \\
\hline 2 & $130(20 \%)$ \\
\hline 3 & $503(77 \%)$ \\
\hline Not assessable & $3(1 \%)$ \\
\hline \multicolumn{2}{|l|}{ Histologic subtype } \\
\hline IDC (NOS ) & $606(92 \%)$ \\
\hline ILC & $15(2 \%)$ \\
\hline Mixed IDC and ILC & $2(1 \%)$ \\
\hline Papillary carcinoma & $2(1 \%)$ \\
\hline Medullary carcinoma & $18(2 \%)$ \\
\hline Metaplastic carcinoma & $9(1 \%)$ \\
\hline Mucinous carcinoma & $1(1 \%)$ \\
\hline \multicolumn{2}{|l|}{ Associated DCIS } \\
\hline Absent & $196(30 \%)$ \\
\hline Low nuclear grade & $9(1 \%)$ \\
\hline Intermediate nuclear grade & $66(10 \%)$ \\
\hline High nuclear grade & $220(34 \%)$ \\
\hline Not mentioned & $162(25 \%)$ \\
\hline \multicolumn{2}{|l|}{ Lymphovascular invasion } \\
\hline Absent & $464(71 \%)$ \\
\hline Present & $189(29 \%)$ \\
\hline \multicolumn{2}{|l|}{ Lymph node status } \\
\hline No nodal metastasis & $320(49 \%)$ \\
\hline Metastasis in 1 to 3 lymph nodes & $129(20 \%)$ \\
\hline Metastasis in 4 or more lymph nodes & $130(20 \%)$ \\
\hline Not available & $74(11 \%)$ \\
\hline \multicolumn{2}{|l|}{ Nuclear pleomorphism } \\
\hline Mild & $3(1 \%)$ \\
\hline Moderate & $227(35 \%)$ \\
\hline Marked & $423(64 \%)$ \\
\hline \multicolumn{2}{|l|}{ Mitotic score (field diameter $0.5 \mathrm{~mm}$ ) ${ }^{\mathrm{a}}$} \\
\hline 1 & $60(9 \%)$ \\
\hline 2 & $137(21 \%)$ \\
\hline 3 & $453(69 \%)$ \\
\hline Not assessable & $3(1 \%)$ \\
\hline \multicolumn{2}{|l|}{ Tubule formation/score } \\
\hline$>75 \%$ tubule formation (score 1) & $15(2 \%)$ \\
\hline $10 \%$ to $75 \%$ tubule formation (score 2 ) & $104(16 \%)$ \\
\hline$<10 \%$ tubule formation (score 3 ) & $534(82 \%)$ \\
\hline \multicolumn{2}{|l|}{ Margins } \\
\hline Infiltrating margin & $548(84 \%)$ \\
\hline Pushing margin & $105(16 \%)$ \\
\hline \multicolumn{2}{|l|}{ Necrosis } \\
\hline Absent & $10(2 \%)$ \\
\hline Present & $643(98 \%)$ \\
\hline \multicolumn{2}{|l|}{ Lymphocytic infiltrates } \\
\hline Mild $(<1 / 3$ tumor area) & $296(45 \%)$ \\
\hline Moderate ( $1 / 3$ to $2 / 3$ tumor area) & $350(53 \%)$ \\
\hline Marked ( $>2 / 3$ tumor area) & $7(2 \%)$ \\
\hline Growth pattern & \\
\hline Trabecular & $290(44 \%)$ \\
\hline Syncytial & $363(56 \%)$ \\
\hline
\end{tabular}

a Among 295 tumors associated with DCIS, 75\% (220/295) DCIS were high nuclear grade.

IDC, infiltrative ductal carcinoma; ILC, infiltrative lobular carcinoma; DCIS, ductal carcinoma in situ; Mitotic activity 0-6/10HPFs (score 1), 7-13/10HPFs (score 2), $\geq 14 / 10 \mathrm{HPFs}$ (score 3 ). 
$77 \%$ ) and $\mathrm{T} 2$ tumors (460 out of $653,70 \%)$. Infiltrative ductal carcinoma was the commonest subtype (606 out of $653,93 \%$ ). A total of 295 tumors were associated with ductal carcinoma in situ, and of these, $220(75 \%)$ were of high nuclear grade. All 15 cases of infiltrative lobular carcinoma were confirmed with E-cadherin-negative immunohistochemical staining.

Tumor size of triple-negative breast cancer was significantly associated with ductal carcinoma in situ, lymphovascular invasion and nodal status $(P=0.000,0.021,0.001)$. Histological grade was correlated with the subtype $(P=0.001)$, whereas lymphovascular invasion was associated with nodal status $(P=0.000)$. There were no statistically significant correlations among other clinicopathological parameters.

\section{Immunohistochemical Findings}

Among the basal antibodies, CK5/6 stained the least (38 out of $653,6 \%$ ) and $34 \beta \mathrm{E} 12$ the most (460 out of
$653,70 \%$ ) cases in our series of 653 triple-negative breast cancers (Figure 5). The results are detailed in Table 3 .

CK5/6 staining intensity correlated with larger tumors $(P=0.033)$ and absence of lymphovascular invasion $(P=0.034)$. Percentage of CK14-positive tumor cells was associated with high-grade tumors $(P=0.05)$, marked nuclear pleomorphism $(P=0.044)$ and a high mitotic score $(P=0.025)$. Both CK17 staining intensity and percentage of positive tumor cells correlated with larger tumors $(P=0.002$, 0.001), high mitotic scores $(P=0.034,0.01)$ and infiltrative margins $(P=0.009,0.001)$. The total percentage of CK17-positive tumor cells correlated with high-grade tumors $(P=0.006)$, marked nuclear pleomorphism $(P=0.000)$ and mild lymphocytic infiltrates $(P=0.002)$. EGFR staining intensity correlated with high histological grade $(P=0.016)$ and marked nuclear pleomorphism $(P=0.013)$ and this also correlated with the total percentage of positive EGFR tumor cells $(P=0.039)$. p63 staining intensity correlated with syncytial growth pattern $(P=0.017)$ and total percentage of p63-positive cells was

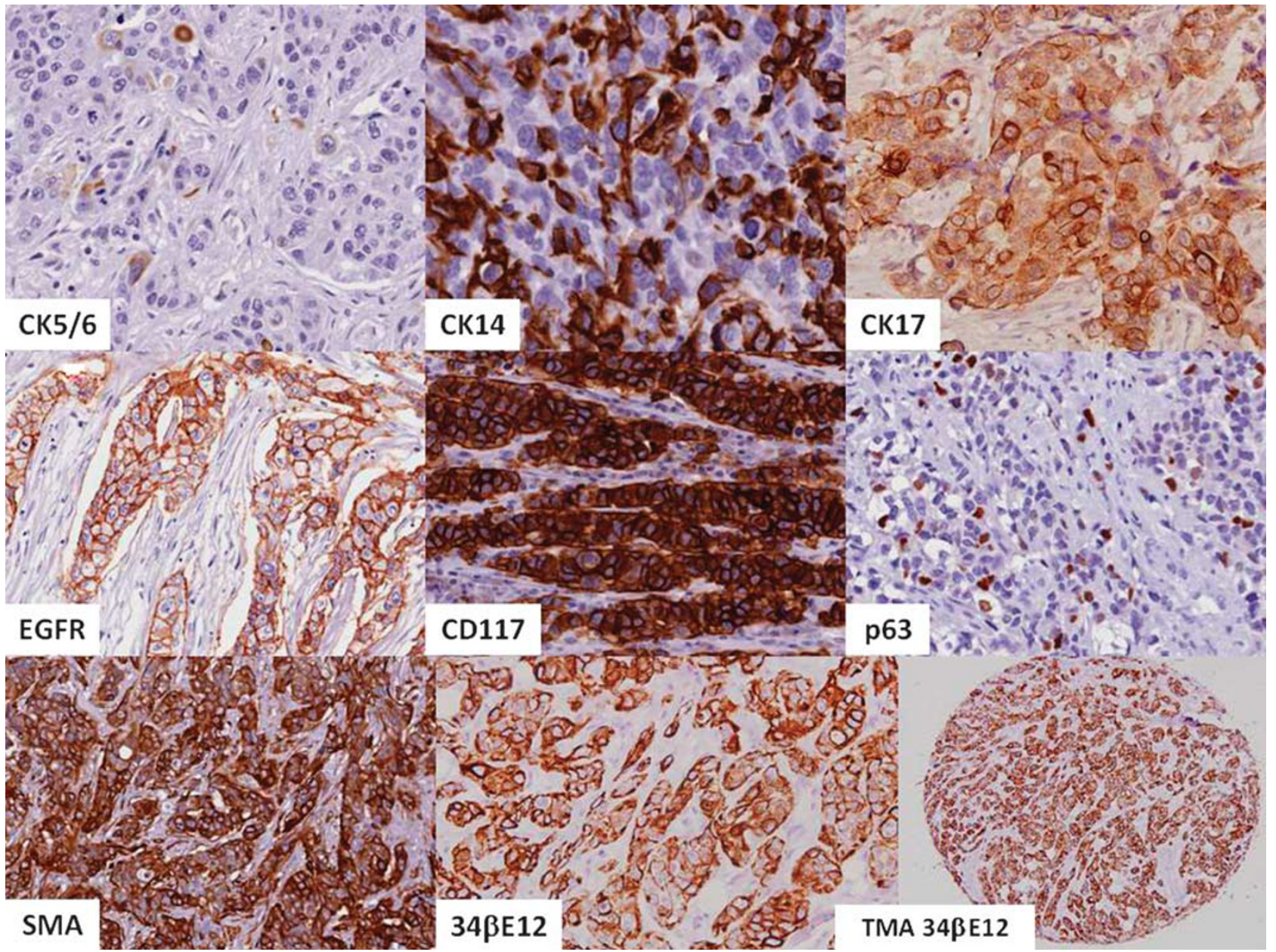

Figure 5 Immunohistochemical staining for CK5/6 with focal patchy reactivity of tumor cells; CK14, CK17, SMA and 34 $\beta$ E12 showed strong cytoplasmic staining of tumor cells; cytoplasmic and membrane positivity for CD117; cytoplasmic membrane reactivity with EGFR; focal nuclear staining with p63. 
Table 3 Immunohistochemical reactivity of basal and other biological markers in 653 cases of TNBC

\begin{tabular}{|c|c|c|c|c|c|}
\hline \multirow[t]{2}{*}{ Basal marker } & \multicolumn{4}{|c|}{ Positive expression } & \multirow[t]{2}{*}{ Negative expression } \\
\hline & $1+$ & $2+$ & $3+$ & Total & \\
\hline CK5/6 & $33(5 \%)$ & $5(1 \%)$ & - & $38(6 \%)$ & $615(94 \%)$ \\
\hline CK14 & $55(8 \%)$ & $69(11)$ & 187 (29\%) & $311(48 \%)$ & $342(52 \%)$ \\
\hline CK17 & $276(42 \%)$ & $30(5 \%)$ & $23(3 \%)$ & $329(50 \%)$ & $324(50 \%)$ \\
\hline EGFR & $159(24 \%)$ & $24(4 \%)$ & $15(2 \%)$ & $198(30 \%)$ & $455(70 \%)$ \\
\hline CD117 & $238(36 \%)$ & $35(6 \%)$ & $19(3 \%)$ & $293(45 \%)$ & $361(55 \%)$ \\
\hline p63 & $101(16 \%)$ & $27(4 \%)$ & $13(2 \%)$ & $141(22 \%)$ & $512(78 \%)$ \\
\hline SMA & $44(7 \%)$ & $24(4 \%)$ & $96(14 \%)$ & $164(25 \%)$ & $489(75 \%)$ \\
\hline $34 \beta \mathrm{E} 12$ & $236(36 \%)$ & $112(17 \%)$ & $112(17 \%)$ & $460(70 \%)$ & $193(30 \%)$ \\
\hline
\end{tabular}

related to marked nuclear pleomorphism $(P=0.028)$. CD117 staining intensity was associated with lymphovascular invasion $(P=0.014)$, infiltrative margin $(P=0.001)$, presence of necrosis $(P=0.014)$ and syncytial growth pattern $(P=0.029)$, whereas the total percentage of CD117-positive tumor cells was related to larger tumors $(P=0.003)$, lymphovascular invasion $(P=0.002)$, high mitotic score $(P=0.001)$ and infiltrative margin $(P=0.0 .03)$. The proportion of SMA-positive tumor cells correlated with high mitotic score $(P=0.014)$. The total percentage of $34 \beta$ E12-positive tumor cells correlated with marked nuclear pleomorphism $(0.022)$ and syncytial growth pattern $(P=0.025)$, whereas its staining intensity was related to the presence of necrosis $(P=0.03)$. No other significant correlations were found.

\section{Immunohistochemical Expression Compared against Molecular Subtypes of 61 Invasive Ductal Carcinomas categorized through Expression Profiling}

In the group of 61 invasive ductal carcinomas classified through expression profiling, $34 \beta$ E12 is the most sensitive (sensitivity $70 \%$ ) in detecting the basal phenotype; however, it showed less specificity (specificity $55 \%$ ). There was a trend for single markers with high specificity to be accompanied by low sensitivity as shown in Table 4 . When a combination of markers was evaluated immunohistochemically, there was an improved diagnostic discrimination between the basal versus cerbB2 and luminal subtypes categorized on expression arrays. A panel of two protein markers confirmed basal-type breast cancer with higher sensitivity and specificity than did a single marker (Table 5). A high sensitivity and specificity could be shown using a combination of three markers (a triple panel) to confirm basal protein immunophenotypic expression among cases categorized as such on expression profiling (Table 6). When we examined the receiveroperating curves to search for criteria providing optimal discrimination, we found that there was a left shift, as combinations of two or three immuno-
Table 4 Sensitivity and specificity of immunohistochemistry for individual markers in determining a basal phenotype in 61 infiltrative ductal carcinomas subtyped on expression profiling

\begin{tabular}{lcccc}
\hline Marker & $\begin{array}{c}\text { Sensitivity } \\
(\%)\end{array}$ & $\begin{array}{c}\text { Specificity } \\
(\%)\end{array}$ & AUC & P-value \\
\hline CK14 & 46 & 88 & 0.672 & 0.022 \\
CK17 & 46 & 88 & 0.672 & 0.022 \\
CD117 & 61 & 88 & 0.771 & 0.000 \\
EGFR & 57 & 94 & 0.541 & 0.582 \\
P63 & 14 & 94 & 0.743 & 0.001 \\
SMA & 29 & 91 & 0.597 & 0.193 \\
34 $\beta$ E12 & 86 & 55 & 0.701 & 0.007 \\
\hline
\end{tabular}

${ }^{\mathrm{a}} \mathrm{AUC}$, area under the ROC curve.

Table 5 Sensitivity and specificity of immunohistochemistry for a combination of two markers in determining a basal phenotype in 61 infiltrative ductal carcinomas subtyped on expression profiling

\begin{tabular}{|c|c|c|c|c|}
\hline $\begin{array}{l}\text { Two marker } \\
\text { combination }\end{array}$ & $\begin{array}{c}\text { Sensitivity } \\
(\%)\end{array}$ & $\begin{array}{c}\text { Specificity } \\
(\%)\end{array}$ & $A U C^{\mathrm{a}}$ & $\mathrm{P}$-value \\
\hline $\mathrm{CK} 14,34 \beta \mathrm{E} 12$ & 80 & 89 & 0.772 & 0.000 \\
\hline CK17, 34 $\beta$ E12 & 80 & 85 & 0.767 & 0.000 \\
\hline EGFR, $34 \beta$ E12 & 88 & 95 & 0.837 & 0.000 \\
\hline CD117, 34 $\beta$ E12 & 88 & 89 & 0.817 & 0.000 \\
\hline
\end{tabular}

${ }^{\mathrm{a}}$ AUC, area under the ROC curve.

Table 6 Sensitivity and specificity of immunohistochemistry for a combination of three markers in determining a basal phenotype in 61 infiltrative ductal carcinomas subtyped on expression profiling

\begin{tabular}{lcccc}
\hline Three marker panel & $\begin{array}{c}\text { Sensitivity } \\
(\%)\end{array}$ & $\begin{array}{c}\text { Specificity } \\
(\%)\end{array}$ & AUC & P-value \\
\hline CK14, CK17, EGFR & 44 & 100 & 0.838 & 0.000 \\
CK14, CK17, 34 $\beta$ E12 & 80 & 94 & 0.797 & 0.000 \\
CK14, EGFR, 34 3 E12 & 78 & 100 & 0.860 & 0.000 \\
CK17, EGFR, 34 $\beta$ E12 & 75 & 94 & 0.860 & 0.000
\end{tabular}

${ }^{a}$ AUC, area under the ROC curve. 

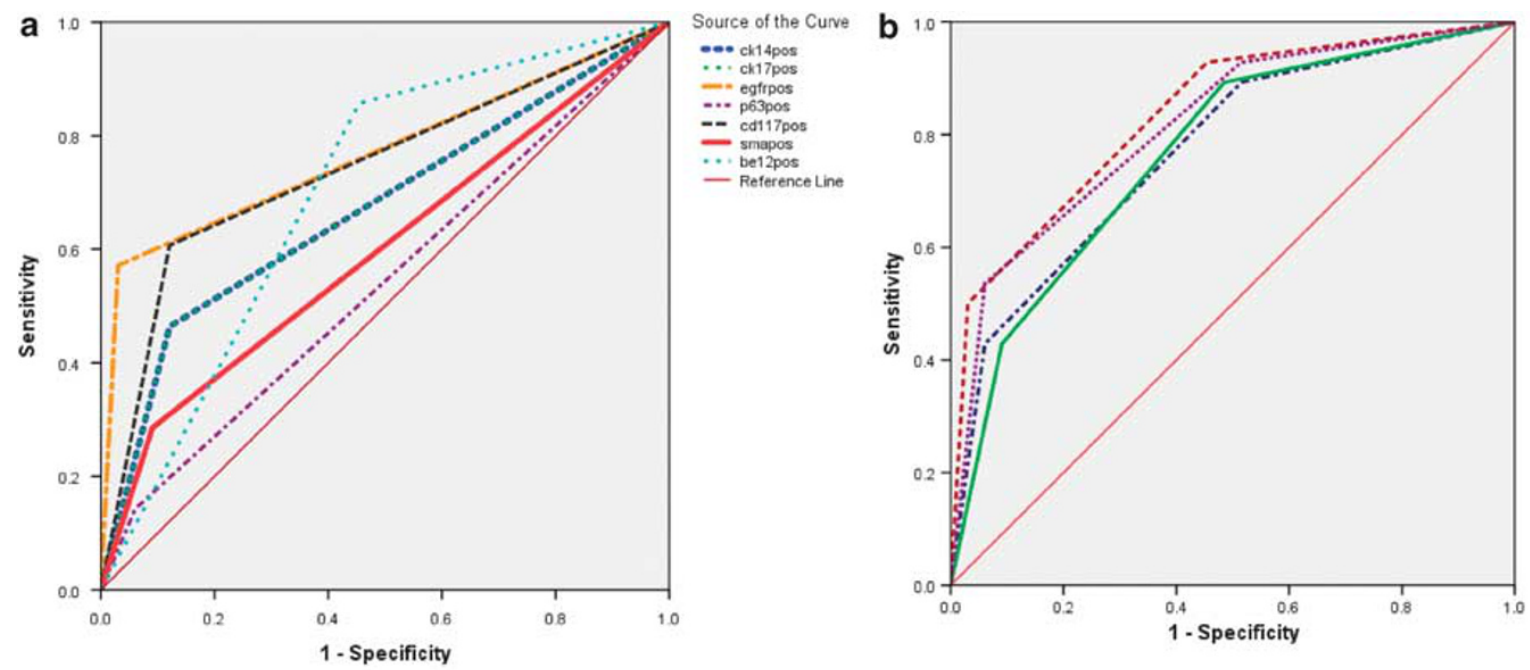
-.. ck14be12 egfrbe12
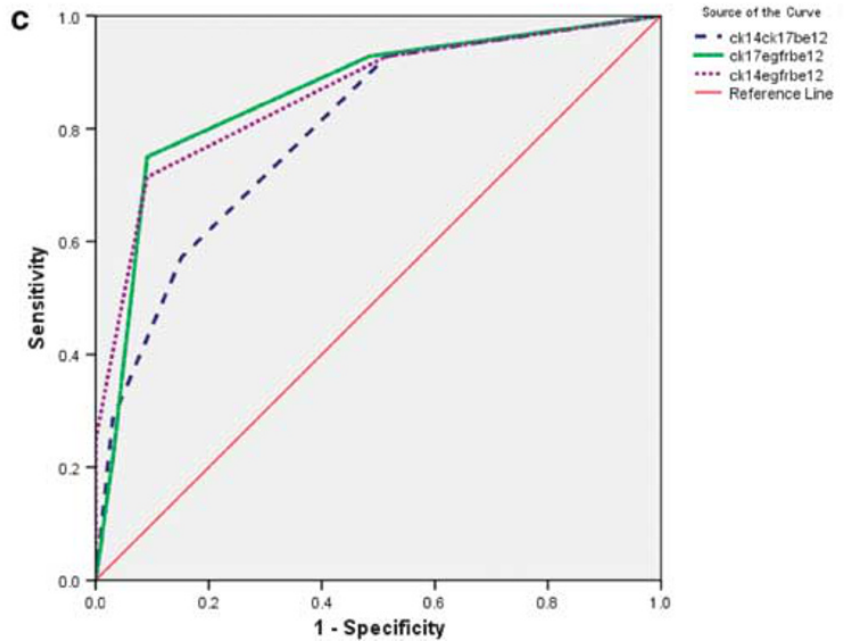

Figure 6 (a) ROC curves for individual immunohistochemical markers. Although they were aligned to the left of the reference line, they remained relatively close to the diagonal reference line. (b) ROC curves for a combination of two immunohistochemical markers (double panel) shifted to the left compared with the curves of the single markers. (c) ROC curves for a combination of three immunohistochemical markers shifted to the left compared with the curves of the single and double immunomarker panels.

Table 7 Sensitivity and specificity of immunohistochemistry for a combination of four markers in determining a basal phenotype in 61 infiltrative ductal carcinomas subtyped on expression profiling

\begin{tabular}{lcccc}
\hline Four markers panel & $\begin{array}{c}\text { Sensitivity } \\
(\%)\end{array}$ & $\begin{array}{c}\text { Specificity } \\
(\%)\end{array}$ & AUC & $\begin{array}{c}\text { P- } \\
\text { value }\end{array}$ \\
\hline CK14, CK17, EGFR, p63 & $86 \%$ & $73 \%$ & 0.863 & 0.000 \\
CK14, CK17, EGFR, CD117 & $86 \%$ & $73 \%$ & 0.863 & 0.000 \\
CK17, EGFR, p63, CD117 & $86 \%$ & $76 \%$ & 0.859 & 0.000 \\
CK17, EGFR, p63, SMA & $79 \%$ & $79 \%$ & 0.813 & 0.000 \\
\hline
\end{tabular}

${ }^{\mathrm{a}} \mathrm{AUC}$, area under the ROC curve.

histochemical markers were used to detect basal phenotype breast cancers as shown in Figures 6a-c, indicating high sensitivity and specificity. This documentation was supported by the area under the receiver-operating characteristic curve values and by significant ' $P$ '-values (Tables 5 and 6 ). When four markers were studied in combination, specificity was decreased (Table 7). On the basis of the findings, it would seem that a triple panel of CK14, EGFR and $34 \beta$ E12 provided the best specificity $(100 \%)$ with reasonable sensitivity $(78 \%)$, and applying this panel to our 653 cases, revealed 549 $(84 \%)$ expressing the basal phenotype.

\section{Discussion}

The incidence of triple-negative breast cancer in our population stands at $11 \%$, which is within the range of $10 \%-16 \%$ generally reported in the literature. ${ }^{20}$ There are scant data on triple-negative breast cancer in Asian women. In Japan, the triple-negative subtype accounted for $15.5 \%$ of breast cancers derived from surveillance data of the Registration Committee of the Japanese Breast Cancer Society, with a predominance of postmenopausal women 
whose cancers were diagnosed at more advanced stages with more frequent nodal metastases. ${ }^{24}$ In another Asian cohort of 683 Korean women with breast cancers, triple-negative breast cancer formed $19.9 \%$ (136 cases) of these tumors, which seems higher than that reported in other studies. ${ }^{25} \mathrm{~A}$ similar result was obtained with a smaller group of 116 Chinese cases, in which 22 (19\%) were triple negative. $^{26}$ With regard to the relationship with basal-like breast cancers, an investigation of 793 Japanese patients with breast cancer reported that only $8 \%$ were basal like with aggressive biological characteristics and a significantly poorer prognosis. ${ }^{27}$ This could be potentially extrapolated to imply that basal-like breast cancers constitute about half of the proportion of triple-negative breast cancers in Japanese women, supporting the premise that these tumors are not synonymous, although the definition and methodology used to delineate the basaloid phenotype will obviously affect the rates. In our study, using a combination of three markers (CK14, EGFR and $34 \beta \mathrm{E} 12$ ), $84 \%$ of our triplenegative breast cancers comprised basal-like tumors, which is at the higher end of the range of $56-84 \%$ reported in the literature of triple-negative breast cancers expressing basal cytokeratins and EGFR. ${ }^{20}$ The ethnic distribution of women in our study did not differ significantly from that observed in our country, in which breast cancers are diagnosed in 83.7\% Chinese, 9.6\% Malay and 5\% Indian women. ${ }^{28}$

Among our cases, the mean and median age of women harboring triple-negative breast cancer was in the early postmenopausal phase of 52 years, and the incidence of women who were 40 years or less among this cohort was not different from the overall rate $(15 \%)$ of breast cancer in this age group documented in our population, suggesting that younger women did not represent a significantly higher proportion of those with triple-negative breast cancer. ${ }^{28}$ Although this is in keeping with Japanese data that indicated a predominance of postmenopausal women among their triple-negative cases, ${ }^{24}$ it differs from that generally reported in which triple negativity affects younger patients under 50 years of age, ${ }^{20}$ as well as from data from the Korean study. ${ }^{25} \mathrm{~A}$ recent study that compared triple-negative breast cancers in women from Vietnam and the United States found that Vietnamese cases were significantly smaller tumors, predominantly ductal in phenotype, less often grade 3 , with fewer metaplastic and no medullary subtypes, as compared with those diagnosed in the United States. $^{29}$

The pathological characteristics in our series confirmed a morphologically aggressive phenotype, with the majority of cases being grade 3 infiltrative ductal carcinomas that measured $>20 \mathrm{~mm}$ in size, with frequent histological identification of necrosis. When ductal carcinoma in situ was present, it was predominantly of high nuclear grade. Although many triple-negative breast cancers have been described to have rounded pushing margins, we found that our cases with such an appearance formed a minority of only $16 \%$. Similarly, marked lymphocytic infiltrates were rare $(2 \%)$, and although a syncytial growth pattern was seen in slightly over half of the cases (56\%), a significant number showed trabecular appearances (44\%) observed in conventional infiltrating ductal carcinomas. Unusually, a few tumors of special subtypes were encountered among the triple-negative breast cancers, suggesting that triple negativity can occur in all histological subtypes of breast cancer with possible implications on their pathogenesis, progression and prognosis. Unlike Dent et $a l,{ }^{9}$ who found no relationship between the tumor size of triple-negative breast cancer and nodal metastasis, our cases showed an increasing incidence of axillary lymph node metastases with enlarging tumor size.

When comparisons were made between immunohistochemical detection of individual biological markers and pathological parameters, there was in general an association of protein expression with aggressive histological features. This is in keeping with our earlier study in which we found that expression of basal keratins in breast cancers of young women correlated with adverse pathological parameters. ${ }^{30}$ Hence, apart from inherent challenges to effective therapies for triple-negative breast cancers that are devoid of currently known therapeutic targets, the expression of basal keratins and other biological markers evaluated in this study may confer added aggressive qualities. The finding of SMA positivity in $25 \%$ of our cases is not surprising. SMA, similar to p63, are myoepithelial markers, both of which have been reported to be expressed in some basal-like breast cancers. Their relatively infrequent documentation, however, has argued against a myoepithelial origin of these tumors. ${ }^{31}$

The relationship between triple-negative and basal-like breast cancer has been discussed and debated at length, and there now seems to be some clarification of the earlier confusion that enveloped these two closely related yet different entities. ${ }^{20,32,33}$ It is recognized that both triple-negative and basallike breast cancers share many similar characteristics. Using microarray-based expression analysis, basal-like breast cancers are mostly triple negative, and vice versa. However, it has been found that 15$45 \%$ of basal-like breast cancers defined on expression profiling express either ER, PR or cerbB2, and only $56-84 \%$ of triple-negative breast cancers manifest basal cytokeratins and EGFR. ${ }^{20}$ It has been reported that patients with triple-negative breast cancer expressing a basal phenotype had significantly shortened disease-free survival than those devoid of basal expression. ${ }^{11,12}$ Interestingly, Fulford et $a l^{5}$ documented a better prognosis in patients whose basal-like cancers possessed a diffuse expression of cytokeratin 14. More recently, Liu et $a l^{34}$ discovered worse disease-free and overall survivals 
in a series of 112 triple-negative breast cancers that disclosed positive staining for CK5/6 or CK17.

Although expression profiling represents the gold standard in molecular subclassification of breast cancer, it is not a tool that would be readily available for routine use in many laboratories, taking into consideration its prohibitive cost and technical difficulties in achieving optimal results with paraffinized material. It is thus useful to determine immunohistochemical surrogates that can be applied to standard paraffin sections that could accurately indicate the presence of a basal phenotype among triple-negative breast cancers.

Analyzed against a group of earlier profiled breast cancers using expression microarrays, we obtained a specificity of $100 \%$ and a sensitivity of $78 \%$ in detecting basal-like cancer, for a triple protein immunohistochemical combination of CK14, EGFR and $34 \beta \mathrm{E} 12$, which exceeds the specificity and sensitivity that could be achieved with dual or four marker panels. Using this immunohistochemical selection, $84 \%$ of our 653 triple-negative breast cancers would be considered basal-like. Nielsen et $a l^{3}$ proposed using negative ER and cerbB2, with a positive expression of CK5/6 and EGFR, to define basal-like cancers, and reported $100 \%$ specificity and $76 \%$ sensitivity. Our results with CK5/6, however, were disappointing, as it decorated only $6 \%$ of triple-negative breast cancers when evaluated individually. We believe that our recommended panel from this study will be efficacious in both cost and technique, as these antibodies are familiar and routinely used in the majority of laboratories. $34 \beta \mathrm{E} 12$ has the advantage of being a basal cell cocktail of high molecular weight cytokeratins 1,4 , 10 and 14, thereby incorporating several keratin forms into one antibody cocktail. The inclusion of EGFR may potentially allow the exploration of the possibility of being a therapeutic target if overexpressed, and CK14 has already been described as defining a better prognosis when exhibiting a diffuse pattern of staining in basal-like breast cancers. ${ }^{32}$

In summary, our study documents clinicopathological features of 653 triple-negative breast cancers derived from a large cohort of 5903 invasive breast carcinomas diagnosed in our department over 14 years from 1994 to 2007, affirming their generally more adverse histological characteristics. Immunohistochemical expression of basal keratins (CK5/6, CK14, CK17, 34 $\beta$ E12), EGFR, CD117, p63 and SMA was evaluated, and a triple panel of CK14, EGFR and $34 \beta$ E12 seems to be the most specific and sensitive in defining basal-like breast cancers among triplenegative breast cancers. Although progress continues to be sought and made in the management of these therapeutically challenging breast tumors, understanding their link with basal-like cancers may provide not only clues to their biology but also offer potential targets that can enhance and improve their treatment options.

\section{Acknowledgement}

This study is supported by grants from the Department of Clinical Research, Singapore General Hospital (DCR/P26/2008) and from the Singapore Cancer Syndicate (MS0004, MS4R).

\section{Disclosure/conflict of interest}

The authors declare no conflict of interest.

\section{References}

1 Sørliea T, Charles M, Peroua CM, et al. Gene expression patterns of breast carcinomas distinguish tumor subclasses with clinical implications. Proc Natl Acad Sci USA 2001;98:10869-10874.

2 Brenton JD, Carey LA, Ahmed AA, et al. Molecular classification and molecular forecasting of breast cancer: ready for clinical application? J Clin Oncol 2005;23:7350-7360.

3 Nielsen TO, Hsu FD, Jensen K, et al. Immunohistochemical and clinical characterization of the basal-like subtype of invasive breast carcinoma. Clin Cancer Res 2004;10:5367-5374.

4 Van de Rijn M, Perou CM, Tibshirani R, et al. Expression of cytokeratins 17 and 5 identifies a group of breast carcinomas with poor clinical outcome. Am J Pathol 2002;161:1991-1996.

5 Fulford LG, Reis-Filho JS, Ryder K, et al. Basal-like grade III invasive ductal carcinoma of the breast: patterns of metastasis and long-term survival. Breast Cancer Res 2007;9:R4.

6 Hicks DG, Short SM, Prescott Nl, et al. Breast cancers with brain metastases are more likely to be estrogen receptor negative, express the basal cytokeratin CK5/6, and overexpress HER2 or EGFR. Am J Surg Pathol 2006;30:1097-1104.

7 Rouzier R, Perou CM, Symmans WF, et al. Breast cancer molecular subtypes respond differently to preoperative chemotherapy. Clin Cancer Res 2005;11:5678-5685.

8 Carey LA, E Dees C, Sawyer L, et al. The triple negative paradox: primary tumor chemosensitivity of breast cancer subtypes. Clin Cancer Res 2007;13:2329-2334.

9 Dent R, Trudeau M, Pritchard KI, et al. Triple negative breast cancer: clinical features and patterns of recurrence. Clin Cancer Res 2007;13:4429-4434.

10 Haffty BG, Yang O, Reiss M, et al. Locoregional relapse and distant metastasis in conservatively managed triple negative early stage breast cancer. J Clin Oncol 2006;24:5652-5657.

11 Rakha EA, El-Sayed ME, Green AR, et al. Prognostic markers in triple negative breast cancer. Cancer 2007;109:25-32.

12 Tischkowitz M, Brunet J-S, Bégin LR, et al. Use of immunohistochemical markers can refine prognosis in triple negative breast cancer. BMC Cancer 2007;7:134.

13 Bauer KR, Brown M, Cress RD, et al. Descriptive analysis of estrogen receptor (ER)-negative, progesterone receptor (PR)-negative, and HER2- invasive breast cancer, the so-called triple-negative phenotype: a population-based study from the California Cancer Registry. Cancer 2007;109:1721-1728.

14 Harris LN, Broadwater G, Lin NU, et al. Molecular subtypes of breast cancer in relation to paclitaxel 
response and outcomes in women with metastatic disease:results from CALGB 9342. Breast Cancer Res 2006;8:R66.

15 Morris GJ, Naidu S, Topham AK, et al. Differences in breast carcinoma characteristics in newly diagnosed African-American and Caucasian patients: a singleinstitution compilation compared with the National Cancer Institute's Surveillance, Epidemiology, and end results database. Cancer 2007;110:876-884.

16 Calza S, Hall P, Auer G, et al. Intrinsic molecular signature of breast cancer in a population-based cohort of 412 patients. Breast Cancer Res 2006;8:R34.

17 Sotiriou C, Neo SY, McShane LM, et al. Breast cancer classification and prognosis based on gene expression profiles from a population-based study. Proc Natl Acad Sci USA 2003;100:10393-10398.

18 Jumppanen M, Gruvberger-Saal S, Kauraniemi P, et al. Basal-like phenotype is not associated with patient survival in estrogen receptor-negative breast cancers. $\mathrm{N}$ Engl J Med 2006;355:560-569.

19 Brennan DJ, Gallagher WM. Prognostic ability of a panel of immunohistochemistry markers-retailoring of an old solution. Breast Cancer Res 2008;10:102-104.

20 Reis-Filho JS, Tutt ANJ. Triple negative tumours: a critical review. Histopathology 2008;52:108-118.

21 NHS Cancer Screening Programmes and The Royal College of Pathologists. Pathology Reporting of Breast Disease. NHSBSP: London, 2005;41-87; NHSBSP Publication no. 58.

22 Tan BKT, Tan LK, Yu K, et al. Clinical validation of a customized multiple signature microarray for breast cancer. Clin Cancer Res 2008;14:461-469.

23 Zweig MH, Campbell G. Receiver-operating characteristic (ROC) plots: a fundamental evaluation tool in clinical medicine. Clin Chem 1993;39:561-577.

24 Iwase H, Kurebayashi J, Tsuda H, et al. Clinicopathological analyses of triple negative breast cancer using surveillance data from the Registration Committee of the Japanese Breast Cancer Society. Breast Cancer 2009, May 23 (E-pub ahead of print).

25 Rhee J, Han SW, Oh DY, et al. The clinicopathologic characteristics and prognostic significance of triplenegativity in node-negative breast cancer. BMC Cancer 2008;8:307.

26 Tian XS, Cong MH, Zhou WH, et al. Clinicopathologic and prognostic characteristics of triple-negative breast cancer. Onkologie 2008;31:610-614.

27 Kurebayashi J, Moriya T, Ishida T, et al. The prevalence of intrinsic subtypes and prognosis in breast cancer patients of different races. Breast 2007;16(Suppl 2): S72-S77.

28 Seow A, Koh WP, Chia KS, et al. Trends in cancer incidence in Singapore 1968-2002. Singapore Cancer Registry Report No. 62004.

29 Williams DJ, Cohen C, To TV, et al. Triple-negative breast carcinoma in women from Vietnam in the United States: characterization of differential maker expression by tissue microarray. Hum Pathol 2009;40:1176-1181.

30 Chen MHS, Yip GWC, Tse GMK, et al. Expression of basal keratins and vimentin in breast cancers of young women correlates with adverse pathologic parameters. Mod Pathol 2008;21:1183-1191.

31 Livasy CA, Karaca G, Nanda R, et al. Phenotypic evaluation of the basal-like subtype of invasive breast carcinoma. Mod Pathol 2006;19:264-271.

32 Da Silva L, Clarke C, Lakhani SR. Demystifying basal-like breast carcinomas. J Clin Pathol 2007;60: 1328-1332.

33 Rakha EA, Ellis IO. Triple-negative/basal-like breast cancer: review. Pathology 2009;41:40-44.

34 Liu ZB, Jiong W, Ping B, et al. Basal cytokeratin expression in relation to immunohistochemical and clinical characterization in breast cancer patients with triple-negative phenotype. Tumori 2009;95: 53-62. 